\title{
Effet de la substitution partielle de la farine de blé par la purée de banane plantain (Musa AAB) bien mûre sur la qualité des produits de pâtisserie
}

\author{
Augustin K. YAO ${ }^{1}$, Djary M. KOFFI' ${ }^{2}$ Zaouli B. IRIÉ ${ }^{1}$ and Sébastien L. NIAMKE ${ }^{2}$ \\ 1 Laboratoire de Chimie-Technologie, Centre National de Recherche Agronomique (CNRA), BP 31 Bingerville, \\ Côte d'Ivoire ; 'Laboratoire de Biotechnologies, Filière Biochimie-Microbiologie de l'Unité de Formation et de \\ Recherche Biosciences de I'Université Félix Houphouët-Boigny, 22 BP 582 Abidjan, Côte d'Ivoire. \\ *Corresponding author e-mail: djaryss@yahoo.fr; Tel : (225) 07028006
}

Original submitted in on $8^{\text {th }}$ October 2014. Published online at www.m.elewa.org on 31st October 2014. http://dx.doi.org/10.4314/jab.v82i1.12

\section{RESUME}

Objectif : La banane plantain, troisième production vivrière en Côte d'Ivoire, est une denrée saisonnière sujette à d'importantes pertes post récolte évaluées à environ $30 \%$. Ces pertes surviennent au bout d'un phénomène de murissement rapide (entre 5 et 9 jours) de la banane mature récoltée. Pour contribuer à lever la contrainte de mauvaise conservation et aussi améliorer la valorisation de la banane plantain, les cultivars Affoto et Orishélé ont été expérimentés pour la production de pulpe séchée puis la fabrication de gâteaux.

Méthodologie et résultats: La pulpe séchée a été obtenue en trois journées de séchage dans un séchoir solaire type NRI et Conditionnée dans des sachets de polyéthylène de 80 microns d'épaisseur, à température ambiante. Les gâteaux ont été préparés à l'aide d'appareils domestiques selon des formulations mettant en œuvre différentes proportions farine de blé et de pulpe séchée de banane rehumidifiée. Différentes méthodes standard ont permis d'effectuer l'analyse microbiologique de la pulpe séchée et de déterminer les caractéristiques biochimiques et organoleptiques des gâteaux fabriqués. Le profil microbiologique de la pulpe séchée et conditionnée dans des sachets de polyéthylène de 80 microns d'épaisseur, est resté constant pendant 12 mois, à température ambiante. Lorsque la proportion de purée de banane augmente le gâteau devient moins énergétique, plus dense et plus tendre. La formule 30/70 (farine de blé / purée de plantain) a été retenue comme formule type pour la préparation du gâteau de plantain, compte tenu des préférences organoleptiques (goût, arôme, aspect, textures...) et également des caractéristiques biochimiques.

Conclusion et applications potentielles: Ces gâteaux présentent un avantage certain, car ils sont faits avec des ingrédients naturels sans sucre ajouté et ont une bonne qualité gustative. La purée de pulpe de plantain bien mûr pourrait être recommandée aux industries locales dans la fabrication des produits de pâtisserie de haute qualité. La production de la pulpe séchée peut se faire à moindre coût.

Mots clés : Substitution partielle, Farine de blé, Pulpe séchée, Purée de banane plantain Musa AAB, Gâteaux de plantain

Effect of partial substitution of wheat flour with mashed ripe plantain (Musa $A A B$ ) on pastry products quality

\section{ABSTRACT}

Objective: Plantain, third food production in Côte d'Ivoire, is a seasonal commodity subject to significant post harvest losses estimated at about $30 \%$. These losses occur after a rapid ripening phenomenon (5 
to 9 days) of the mature banana harvested. To help overcome the stress of poor preservation and improve the development of plantain, cultivars Affoto and Orishélé were tested for the production of dried pulp and making cakes.

Methodology and results: The dried pulp was obtained three days of drying in a solar dryer NRI and packed in polythene bags of 80 microns thickness for 12 months at room temperature. The cakes were prepared using household appliances according formulations implementing different proportions of wheat flour and banana dried pulp re-moistened. Different standard methods have allowed for the microbiological analysis of the dried pulp and to determine the biochemical and organoleptic characteristics of manufactured cakes. Microbiological profile of the dried pulp and packed in polyethylene bags of 80 micron thick remained constant for 12 months at room temperature. When the proportion of mashed banana cake increases the energy becomes less, denser and softer. The 30/70 formula (wheat flour / mashed plantain), was selected as the standard form for the preparation of plantain cake, given the organoleptic preferences (taste, aroma, appearance, texture) and the biochemical characteristics.

Conclusion and findings application: To sum up, banana cakes have a certain advantage because they are made with natural ingredients with no added sugar and have a good flavour. Mashed pulp of ripe plantain could be recommended to local industries in the manufacture of pastry products of high quality. The production of dried pulp can be done cheaply.

\section{INTRODUCTION}

La banane plantain est une importante culture vivrière aussi bien dans le monde qu'en Afrique et plus particulièrement en Côte d'Ivoire par sa production et sa consommation. Elle est essentiellement utilisée pour l'alimentation humaine. Au plan mondial, cette culture occupe le 5 ème rang des productions vivrières, avec une production annuelle estimée à plus de 100000 000 tonnes (Picq et al. 1998 ; FAO, 2009a). Sa production en Afrique est estimée à 24734204 tonnes. L'Ouganda en est le premier producteur mondial avec une production de 9371000 tonnes (FAO, 2009b). En Côte d'lvoire, la banane plantain, dont la production annuelle est d'environ 1555454 tonnes, occupe le 3ème rang des productions vivrières, après l'igname et le manioc (Du croquet, 2002 ; Anonyme 1, 2008). La banane plantain est produite dans les zones souvent éloignées des grands centres de consommation et l'évacuation de la production se fait difficilement (Tano, 1979). Par ailleurs, cette production est saisonnière et la récolte s'étale d'octobre à mars, période au cours de laquelle des pertes évaluées à environ $30 \%$ sont enregistrées, suivie d'une période de faible production, d'avril à septembre (Kuperminc, 1985 ; Sery, 1988). Dans les conditions normales de température ambiante $\left(30^{\circ} \mathrm{C}\right)$, elle mûrit entre 5 et 9 jours après la récolte, si la maturité physiologique est atteinte. Cet intervalle de temps est réduit par l'entreposage défectueux et le manque de soins au cours des diverses manipulations post-récolte (transport, étalage sur les marchés, etc.) (Guillemot, 1976). La banane plantain destinée exclusivement à l'alimentation humaine sert ainsi à la préparation de divers plats traditionnels à consommation immédiate dont les plus importants sont: le foutou, le foufou, l'aloco, l'akpessi, le locloun, le cracro et l'apity (Lassoudière, 1973; Agbo et Coulibaly, 1989). La conservation poste récolte est un facteur limitant pour la disponibilité et la régularité de cette matière première. En effet, les traitements appliqués au plantain au cours de la récolte et l'utilisation post récolte sont effectués de façon traditionnelle. II y a très peu de plantations de bananiers plantains en culture pure (Lassoudière, 1973; Guillenot, 1976; Soudain et al., 1986). La banane plantain est produite dans les zones souvent éloignées des grands centres de consommation et l'évacuation de la production se fait difficilement (Tano, 1979). Par ailleurs, cette production est saisonnière et la récolte s'étale d'octobre à mars, période au cours de laquelle des pertes évaluées à environ $30 \%$ sont enregistrées, suivie d'une période de faible production, d'avril à septembre (Kuperminc, 1985; Sery, 1988). Dans les conditions normales de température ambiante $\left(30^{\circ} \mathrm{C}\right)$, elle mûrit entre 5 et 9 jours après la 
récolte, si la maturité physiologique est atteinte. Cet intervalle de temps est réduit par l'entreposage défectueux et le manque de soins au cours des diverses manipulations post-récolte (transport, étalage sur les marchés, etc.) (Guillemot, 1976). II n'existe pas de véritable méthode de conservation ou de transformation en produits finis ou semi finis permettant un stockage de longue durée de la banane plantain (Soudain et al., 1986). En milieu rural, la pulpe de banane plantain mûr est souvent séchée au soleil et consommée comme friandise, mais, il n'y a pas de disposition pour permettre la conservation de longue durée de ce produit. Cette pulpe séchée, très riche en sucres, se réhydrate rapidement si elle n'est pas emballée et le développement des levures et moisissures entrainent les pourritures qui la rendent impropres à la consommation. En outre, il n'y a pas de structure de transformation industrielle de la banane plantain pour résorber le surplus de production et réduire les pertes. Des études pour la transformation de la banane plantain en produits traditionnels améliorés sont en cours et permettront à terme, la mise au point de farines stabilisées. Ces dernières pourront être utilisées pour la préparation du foutou de banane et autres produits. L'utilisation de la banane plantain mûre pour la fabrication de produits de

\section{MATERIEL ET METHODE}

Matériel végétal: Les bananes plantains utilisées pour nos essais sont les cultivars Affoto (faux corne) et Orishélé de pleine maturité (80 jours). Elles ont été obtenues sur 4 sites en Côte d'Ivoire: la Station Expérimentale d'Anguédédou - Azaguié, à 20 kilomètres d'Abidjan sur l'axe Abidjan Dabou, la Station de La Mé, à 30 kilomètres d'Abidjan sur l'axe Abidjan Alépé, ainsi que sur des plantations de la région de Tiassalé et Taabo, situées à 176 kilomètres au nord d'Abidjan sur l'axe Abidjan Taabo.

Ingrédients : Les ingrédients de base utilisés pour la préparation du gâteau de plantain sont : la farine de blé pâtissière, la purée de banane plantain (pulpe sèche rehumidifiée), l'huile végétale, les œufs et la levure chimique. Le gâteau de banane plantain ne pâtisserie est très peu documentée. Les études de Bonet et al. (2008), Akesowan (2009), Hemeda et Mohamed (2010), Ndife et al. (2011), Chaiya et Pongsawatmanit (2011) et Hussein et al. (2011) ont permis de substituer partiellement la farine de blé avec des farines non panifiables (riz, manioc, légumineuses etc.) pour fabriquer des gâteaux. Ces différentes études ont montré qu'il est possible de substituer la farine de blé jusqu'à $50 \%$, avec d'autres produits, pour obtenir des gâteaux aux caractéristiques similaires à ceux préparés avec $100 \%$ de farine de blé. Des gâteaux à la banane Musa $A A A$ existent, mais ne sont pas très connus. Mepba et al. (2007) ont fabriqué du pain et des biscuits en substituant la farine de blé avec la farine de banane plantain. Les formulations utilisant à 20 et $40 \%$ de farine de banane plantain respectivement pour le pain et les biscuits, ont donné des produits acceptables. Cette étude a pour but d'apporter une valeur ajoutée à la banane plantain mûre périssable. II s'agit de mettre à la disposition des opérateurs de la filière, des méthodes de conservation, facilement réalisables ainsi que des procédés de transformation simples et peu onéreuses, se rapprochant le plus possible des produits traditionnels.

contient pas de sucre ajouté, ni de beurre, celui-ci étant remplacé par de l'huile végétale.

Matériels techniques: Un séchoir solaire, variante du séchoir cadre type NRI, a été fabriqué et utilisé pour le séchage de la pulpe de la banane plantain mûre (Photographie 1).

Pour la confection du gâteau de plantain, les matériels suivants ont été utilisés : une cuisinière à gaz équipée d'un grand four de $85 \mathrm{~cm}$ de longueur, $50 \mathrm{~cm}$ de largeur et $45 \mathrm{~cm}$ de profondeur et muni de deux thermomètres de 50 à $300^{\circ} \mathrm{C}$, un broyeur de marque MOULINEX type super Blender 1 , un batteur mixeur de marque CHROMEX type TGL 1113, une balance de portée $5 \mathrm{~kg}$ de marque SOEHNLE, et des moules en acier revêtues de film antiadhésif. 


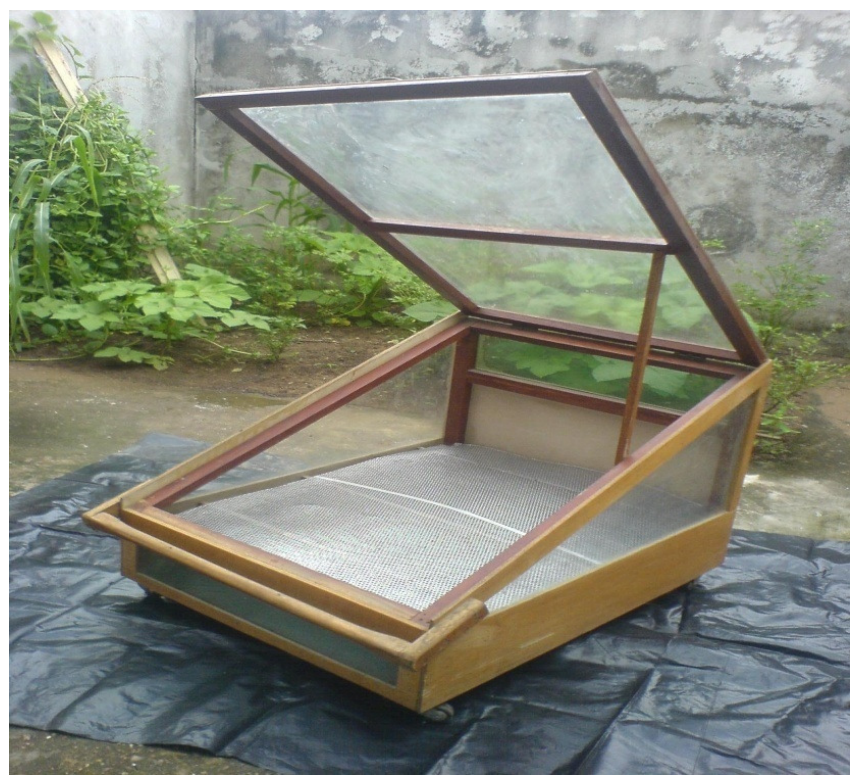

Photographie 1: Séchoir solaire cadre type NRI utilisé pour le séchage de la pulpe de banane plantain Méthodes

Séchage solaire de la pulpe de banane plantain mûre: La banane plantain, uniformément mûre et sans pourriture, a été utilisée pour le séchage. Pour ce faire, les bananes mûres sont épluchées et les pulpes fendues longitudinalement avec des couteaux en métal inoxydable. Une fois la partie intérieure centrale enlevée, la pulpe est découpée en petits morceaux de $1 \mathrm{~cm}$ d'épaisseur qui sont ensuite étalés sur le grillage du séchoir (Photographie 2). Le couvercle recouvert d'un film de polyéthylène de 345 microns d'épaisseur est rabattu pour fermer le séchoir qui est ensuite exposé au soleil pendant $18 \mathrm{~h}$ d'ensoleillement, soit environ trois journées. La pulpe séchée est conditionnée dans des sachets de polyéthylène de 80 microns d'épaisseur, thermo soudé puis stockée à température ambiante dans un endroit sec.

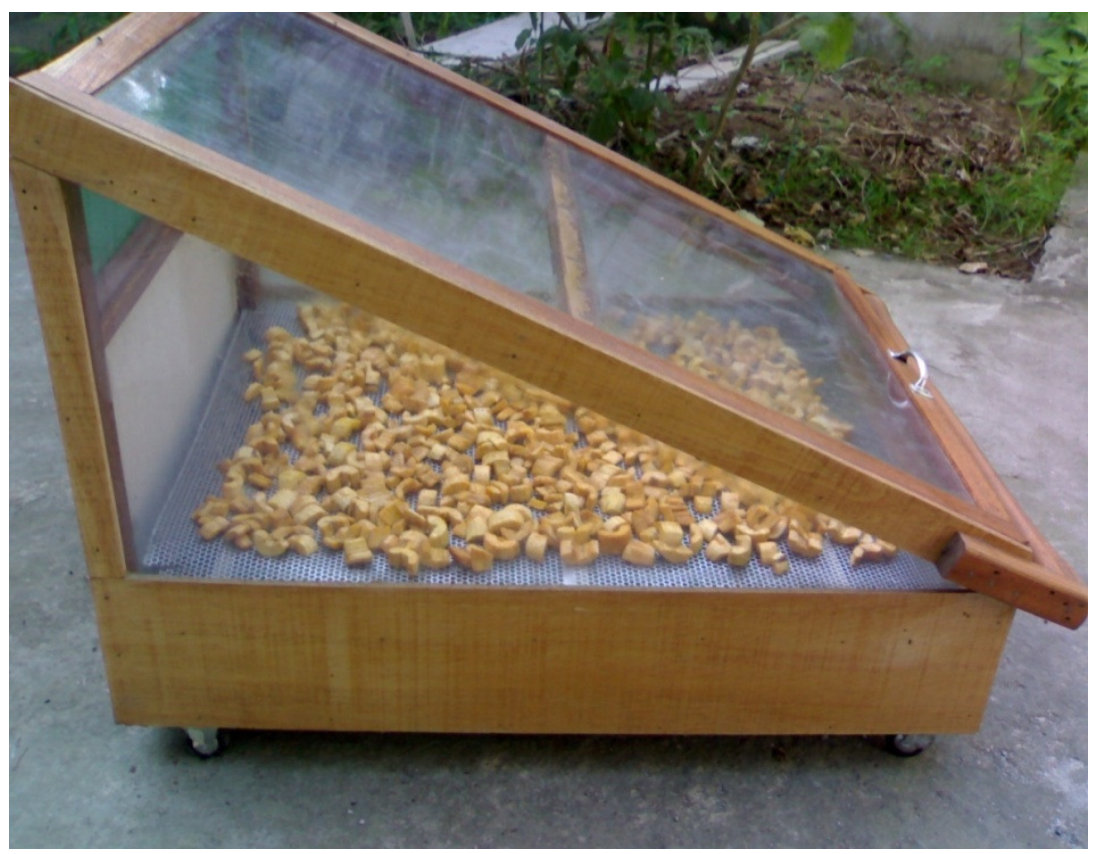

Photographie 2: Séchage de la pulpe de banane plantain bien mûre à l'aide du séchoir solaire cadre type NRI.

Fabrication des gâteaux : La fabrication du gâteau est faite, soit avec la pulpe fraîche bien mûre broyée avec un broyeur mixeur de marque MOULINEX type super Blender I, soit avec la pulpe séchée, réhydratée par trempage dans une quantité d'eau égale à 1,5 fois le poids de pulpe séchée. La pulpe 
séchée réhydratée est ensuite broyée et la purée fine obtenue est utilisée pour la fabrication de gâteaux. Ainsi, des œufs et de l'huile végétale sont ajoutés à la purée de banane plantain, en fonction de la quantité du mélange de farine et de purée de plantain utilisé. Pour un mélange de $1297 \mathrm{~g}$ constitué de 700 $\mathrm{g}$ de purée de banane plantain $(54,32 \%), 300 \mathrm{~g}$ de farine $(23,49 \%), 2$ œufs (100g) $(7,83 \%), 175 \mathrm{~g}$ d'huile végétale $(13,7 \%)$ et $22 \mathrm{~g}$ de levure chimique $(1,72 \%)$. Le mélange de la purée de banane plantain des œufs et de l'huile est soigneusement homogénéisé avec un batteur, pendant 2 à $3 \mathrm{~min}$, suivi de l'addition de la farine panifiable tout en pétrissant pendant $4 \mathrm{~min}$. Enfin $22 \mathrm{~g}$ de levure chimique sont ajoutés et le tout bien mélangée pendant $2 \mathrm{~min}$. A la fin du pétrissage, les moules métalliques de $1600 \mathrm{~cm}^{3}$ de capacité revêtus de film anti adhésif sont remplis et la cuisson se fait dans le four, à partir de $150^{\circ} \mathrm{C}$, en augmentant graduellement la température, pour atteindre, en fin de cuisson $180^{\circ} \mathrm{C}$. La cuisson se fait pendant $1 \mathrm{~h}$ de temps (Turabi et al., 2008).

Analyses microbiologiques: En vue d'évaluer la qualité sanitaire des produits de transformation de la banane plantain, des analyses microbiologiques ont été effectuées sur la pulpe de banane plantain mûre séchée. Les germes suivants ont été déterminés : les germes aérobies mésophiles totaux par la méthode NF ISO 4833, les levures et moisissures par la méthode NF ISO 7954, les salmonelles (NFISO 6579), les micro-organismes indicateurs de contamination fécales Eschérichia coli, coliformes fécaux (NF ISO 08-017), les staphylocoques (NF ISO 6888), les entérobactéries (NF ISO 07402), les aérobies sulfito réducteurs (NF $V$ 08-019) et les coliformes totaux (NF ISO 4832).

Détermination du taux d'humidité: Le taux d'humidité a été déterminé selon la méthode AOAC (1980). Les échantillons sont pesés $\left(P_{0}\right)$ à l'aide d'une balance de précision de type SARTORUIS BP 310S, Gottingen, West Germany. Ils sont séchés dans une étuve de marque (MEMERT, Schwabach West Germany), à $105^{\circ} \mathrm{C}$, pendant $24 \mathrm{~h}$. A la sortie de l'étuve, les échantillons sont refroidis dans un dessiccateur et pesés $\left(P_{1}\right)$; le pourcentage d'humidité a été déterminé par calcul selon, la formule :

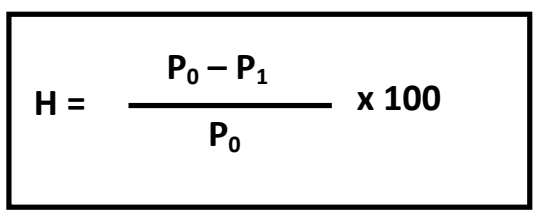

$H: \%$ humidité ; $P_{0}$ : masse échantillon avant séchage ; $P_{1}$ : masse échantillon après séchage.

Dosage des protéines brutes: Le dosage des protéines a été fait selon la méthode de Kjeldahl; cette méthode est basée sur le dosage de l'azote total, qui est ensuite converti en taux de protéines. La mise en œuvre de cette méthode comprend une phase de minéralisation, suivie d'une phase de distillation, et d'une phase de dosage par l'acide sulfurique (BIPEA, 1976). La minéralisation de $1 \mathrm{~g}$ d'échantillon des produits concernés se fait en présence de catalyseur composé de sulfate de cuivre $\left(\mathrm{CuSO}_{4}\right)$ et de sulfate de potassium $\left(\mathrm{K}_{2} \mathrm{SO}_{4}\right)$ dans un digesteur type Buchi 430 (Digestor Germany), pendant $3 \mathrm{~h}$. La distillation est effectuée dans un distillateur type Buchi 320, Germany, après addition de $10 \mathrm{~mL}$ de solution de soude $(\mathrm{NaOH})$ à $40 \%$ au minéralisât. Le distillat est recueilli dans une solution tampon d'acide borique préparée par dissolution de $10 \mathrm{~g}$ d'acide borique dans $1000 \mathrm{~mL}$ d'eau distillée et $11 \mathrm{~mL}$ de mélange d'indicateur coloré constitué de vert de bromocrésol et de rouge de méthyl. Le titrage du distillat se fait avec l'acide chlorhydrique $(\mathrm{HCl}) 0,1$ $\mathrm{N}$. Tous les composés azotés sont dosés avec cette méthode, alors que les protéines représentent $16 \%$ de ces composés; le taux d'azote total a été converti en taux de protéines, en utilisant le facteur 6,25 , soit 100/16. Les taux d'azote total et de protéines brutes sont obtenus avec les formules suivantes :

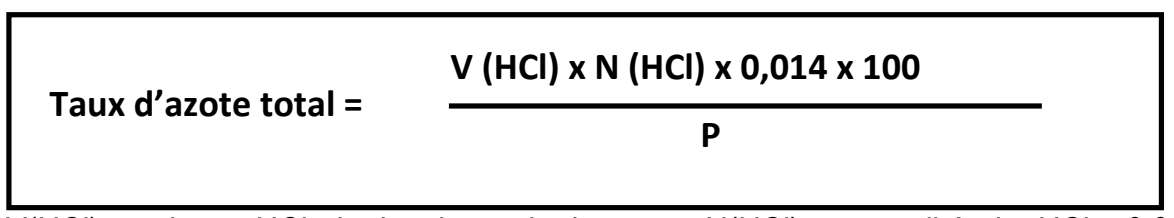

$\mathrm{V}(\mathrm{HCl})$ : volume $\mathrm{HCl}$ de la chute de burette; $\mathrm{N}(\mathrm{HCl})$ : normalité de $\mathrm{HCl} ; 0,014$ : coefficient affecté à la concentration de la solution normale d'azote (14/1000) ; $P$ : poids de l'échantillon 
Taux de protéines brutes $=$ Taux d'azote total x 6,25

Détermination du taux de cendres: Cette détermination a été faite selon la méthode AOAC (1980) ; elle consiste à minéraliser un échantillon de $5 \mathrm{~g}\left(\mathrm{P}_{0}\right)$ à $550^{\circ} \mathrm{C}$ pendant $6 \mathrm{~h}$ dans un four à moufle (NABERTERM, Gmbh LT9/11/B180, Germany), jusqu'à destruction de toutes les matières organiques contenus dans l'échantillon. La pesée $\left(P_{1}\right)$ après refroidissement dans un dessiccateur de la cendre obtenue a permis de déterminer le taux de cendres selon la formule :

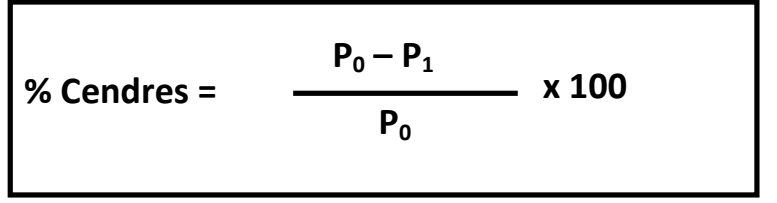

$P_{0}$ : masse échantillon avant minéralisation; $P_{1}$ : masse échantillon après minéralisation

Détermination du taux de matières grasses : Cette détermination est basée sur le fait que les matières grasses sont solubles dans les solvants organiques tels que l'éther de pétrole et l'hexane. La détermination a été faite suivant la méthode du BIPEA (1976), consistant à extraire les matières grasses avec l'hexane, qui est ensuite évaporé, et, le résidu, séché, puis pesé. Nous avons utilisé le SOXTHERM automatic (gerhardt Germany) pour faire l'extraction des matières grasses.

Détermination de la teneur en glucides totaux : Le taux de glucides totaux a été déterminé selon la formule (Bertrand et Thomas, 1910) :

Glucides totaux $=100 \%$ - (\%humidité $+\%$ protéines + \%lipides + \%cendres)

Détermination de la valeur énergétique :

$E$ (calories $)=(4 \times \%$ protéines $)+(4 \times \%$ glucides totaux $)+(9 \times \%$ lipides $)$

La valeur énergétique a été déterminée par calcul, selon la formule de Coleman (1970) utilisant les coefficients d'Atwater et Rosa (1899).

Le volume des gâteaux a été déterminé en multipliant la longueur, la largeur et la profondeur obtenues en mesurant avec une règle; le gâteau démoulé est pesé à l'aide d'une balance de marque Sartorius de portée $2,2 \mathrm{~kg}$ avec une précision de 0,01g. La densité a été obtenue en divisant le poids par le volume.

Evaluation organoleptique: Les échantillons des différentes formulations de gâteau ont été évalués par 30 dégustateurs familiers aux caractéristiques des gâteaux. Le panel de dégustateurs étaient composés de 5 professionnels de pâtisserie et 25 consommateurs. Les échantillons de gâteau ont été codés par des lettres alphabétiques avec deux lettres différentes pour chaque échantillon pour nous permettre de déceler les éventuels résultats erronés qui ont été par la suite éliminés dans le résultat final. L'évaluation sensorielle a été conduite dans une salle bien aérée à température ambiante et tous les échantillons ont été servis en même temps le même jour. II a été demandé aux dégustateurs de noter les critères goût, couleur, arôme, aspect et texture, sur une échelle de 1 à 5 . Les dégustateurs disposaient de l'eau pour se rincer la bouche après la dégustation de chaque échantillon. A l'issu de la dégustation, les notes ont été analysées statistiquement par le test de Newman- Keuls au seuil de $5 \%$ de comparaison des moyennes pour 
déterminer s'il y a des différences significatives entre les différents échantillons.

Analyse statistique des résultats : Les résultats ont été soumis à des analyses de variances (ANOVA) réalisées avec le logiciel Stastica 7.0 en vue de

\section{RESULTATS}

Profil microbiologique de la pulpe de banane plantain mûre séchée et conditionnée: Le profil microbiologique de la pulpe séchée et conditionnée dans des sachets de polyéthylène de 80 microns comparer les moyennes. En cas de différence significative, le test de Newman-Keuls a permis d'identifier les moyennes responsables de la différence observée au seuil de $5 \%$.

d'épaisseur, est resté constant pendant 12 mois, à température ambiante. Durant toute cette période de conservation, ces pulpes séchées ont été trouvées de qualité microbiologique satisfaisante tableau 1.

Tableau 1: Profil microbiologique de la pulpe de banane plantain mûre séchée et conditionnée dans des sachets de polyéthylène de 80 microns d'épaisseur et conservée pendant 12 mois à température ambiante.

\begin{tabular}{|c|c|c|c|c|c|c|}
\hline & \multicolumn{6}{|c|}{ Temps de conservation (mois) } \\
\hline Paramètres & Méthodes & 0 & 3 & 6 & 12 & $\begin{array}{l}\text { Normes } \\
\text { internationales }\end{array}$ \\
\hline $\begin{array}{l}\text { Aérobies } \\
\text { mésophiles totales/g }\end{array}$ & $\begin{array}{l}\text { NF ISO } \\
4833\end{array}$ & $6.10^{3}$ & $6.10^{3}$ & $6.10^{3}$ & $6.10^{3}$ & $3.10^{4}$ \\
\hline Coliformes totaux/g & $\begin{array}{l}\text { NF ISO } \\
4832\end{array}$ & $<10$ & $<10$ & $<10$ & $<10$ & $10^{3}$ \\
\hline Coliformes fécaux/g & $\begin{array}{l}\text { NF ISO } \\
4832 \\
\end{array}$ & $<10$ & $<10$ & $<10$ & $<10$ & 10 \\
\hline E. coli & $\begin{array}{l}\text { NF V } \\
08-017\end{array}$ & $<10$ & $<10$ & $<10$ & $<10$ & \\
\hline $\begin{array}{l}\text { Staphylocoques } \\
\text { dorés/g }\end{array}$ & ISO 6888 & $<10$ & $<10$ & $<10$ & $<10$ & 10 \\
\hline $\begin{array}{l}\text { Anaérobies sulfito- } \\
\text { réducteurs } 46^{\circ} \mathrm{C} / \mathrm{g}\end{array}$ & $\begin{array}{l}\text { NF V } \\
08-019\end{array}$ & $<10$ & $<10$ & $<10$ & $<10$ & $<10$ \\
\hline Entérobactéries/g & $\begin{array}{l}\text { NF ISO } \\
7402\end{array}$ & $<10$ & $<10$ & $<10$ & $<10$ & \\
\hline Salmonelles/g & $\begin{array}{l}\text { NF ISO } \\
6579\end{array}$ & absence & absence & absence & absence & absence \\
\hline Levures & $\begin{array}{l}\text { NF ISO } \\
7954\end{array}$ & $<10$ & $<10$ & $<10$ & $<10$ & $10^{2}$ \\
\hline Moisissures & $\begin{array}{l}\text { NF ISO } \\
7954\end{array}$ & $<10$ & $<10$ & $<10$ & $<10$ & $10^{2}$ \\
\hline
\end{tabular}

Composition biochimique et caractéristiques physiques des gâteaux des différentes formulations: La composition biochimique et les caractéristiques physiques des gâteaux des différentes formulations sont présentées dans le tableau 2. Cette composition varie en fonction des proportions des principaux ingrédients utilisés, que sont la farine et la purée de pulpe de banane plantain. Nous avons observé que les taux de glucides totaux et de protéines diminuent, alors que ceux de l'humidité et des cendres augmentent quand le taux de farine diminue. Le taux de lipides est resté constant, car la même quantité d'huile a été utilisée pour toutes les formulations. Quant aux caractéristiques physiques, le volume et l'apport énergétique baissent quand le taux de farine dans la formulation diminue. A l'inverse, une augmentation du poids et de la densité a été observée. Les gâteaux obtenus sont de couleur jaune orangé et présentent une mie avec une texture feuilletée, à alvéoles régulières, un aspect attrayant, un volume double de pâte de départ, une faible densité et un arôme caractéristique de la banane plantain mûre séchée (Photographie 3). Toutes ces caractéristiques ont été utilisées pour l'évaluation organoleptique des gâteaux. 


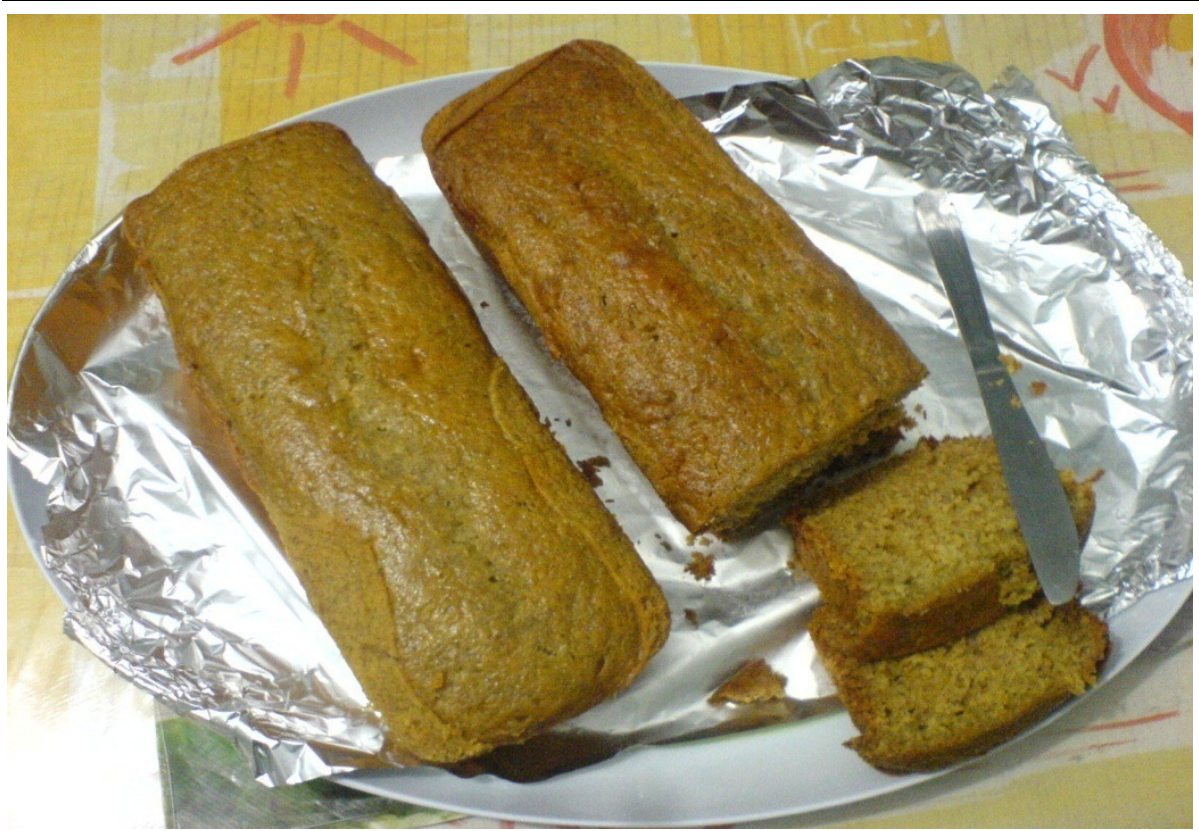

Photographie 3 : Gâteau de banane plantain 30/70

Le taux d'humidité des gâteaux est compris entre 21,3 et $32,2 \%$ pour les différentes formulations. Le taux le plus bas a été obtenu avec le gâteau préparé avec $100 \%$ de farine de blé et le plus élevé avec le gâteau du mélange $80 \%$ de banane plantain et $20 \%$ de farine de blé. Des différences significatives $(p \geq$ 0,05 ) ont été observées entre les gâteaux de $100 \%$ farine de blé et ceux issus du mélange farine de blé et purée de banane plantain. Les taux de protéines, de glucides totaux et de cendres varient respectivement de 12,1 à $9,74 \%$, de 56,9 à $38,9 \%$ et de 2,5 à $3,02 \%$ respectivement pour le gâteau fait avec $100 \%$ farine de blé et celui obtenu avec le mélange $80 \%$ plantain et $20 \%$ farine de blé. Le gâteau préparé avec $100 \%$ de farine, avait un volume plus grand $\left(1650 \mathrm{~cm}^{3}\right)$ que ceux issus des formulations de 75 à $20 \%$ de farine de blé qui varient entre 1635 et $1545 \mathrm{~cm}^{3}$. Une différence significative $(p \geq 0,05)$ a été observée entre les volumes des gâteaux de formulations 100 à $30 \%$ de farine de blé et $20 \%$ de farine de blé. Le poids et la densité des gâteaux varient de 860,5 à $920,5 \mathrm{~cm}^{3}$ et de 0,51 à $0,59 \mathrm{~g} / \mathrm{cm}^{3}$ respectivement, pour les formulations $100 \%$ de farine de blé et $20 \%$ farine de blé et $80 \%$ de banane plantain pour laquelle une différence significative $(p \geq 0,05)$ a été observée pour les deux caractéristiques. Le gâteau fait avec $100 \%$ de farine de blé, était plus énergétique (412 kcal/100 g) alors que ceux des différents mélanges, farine de blé et banane plantain ont des valeurs énergétiques qui varient de 402,35 à $326,32 \mathrm{kcal} / 100 \mathrm{~g}$. 
Yao et al. J. Appl. Biosci. 2014.

Effet de la substitution partielle de la farine de blé par la purée de banane plantain (Musa AAB) bien mûre sur la qualité des produits de pâtisserie

Tableau 2 : Composition biochimique et caractéristiques physiques des gâteaux en fonction des différentes formulations.

$$
\text { Ingrédients Paramètres }
$$

\begin{tabular}{|c|c|c|c|c|c|c|c|c|c|c|}
\hline $\begin{array}{l}\text { Farine de } \\
\text { blé }\end{array}$ & $\begin{array}{ll}\begin{array}{l}\text { Purée } \\
\text { plantain }\end{array} & \text { de } \\
\end{array}$ & Humidité (\%) & Protéines (\%) & $\begin{array}{c}\text { Lipides } \\
(\%)\end{array}$ & $\begin{array}{l}\text { Glucides } \\
\text { totaux (\%) }\end{array}$ & $\begin{array}{c}\text { Cendres } \\
(\%)\end{array}$ & $\begin{array}{c}\text { Volume } \\
\left(\mathrm{cm}^{3}\right)\end{array}$ & Poids (g) & $\begin{array}{l}\text { Densité } \\
\left(\mathrm{g} / \mathrm{cm}^{3}\right)\end{array}$ & $\begin{array}{l}\text { Énergie } \\
\text { Kcal } / 100 \mathrm{~g}\end{array}$ \\
\hline 100 & 0 & $21,3^{a}$ & $12,1^{c}$ & $14,63^{a}$ & $56,90^{c}$ & $2,5^{a}$ & $1650^{b}$ & $860,5^{a}$ & $0,51^{a}$ & $412^{c}$ \\
\hline 75 & 25 & $24,93^{b}$ & $12,0^{c}$ & $14,63^{a}$ & $55,67^{c}$ & $2,63^{a}$ & $1635^{b}$ & $861,67^{a}$ & $0,52^{a}$ & $402,35^{b}$ \\
\hline 60 & 40 & $25,08^{b}$ & $10,57^{b}$ & $14,63^{a}$ & $54,70^{c}$ & $2,67^{a}$ & $1630^{b}$ & $862,5^{a}$ & $0,53^{a}$ & $392,75^{b}$ \\
\hline 50 & 50 & $27,1^{b}$ & $10,92^{b}$ & $14,62^{\mathrm{a}}$ & $50,77^{b}$ & $2,74^{a}$ & $1632^{b}$ & $901,00^{b}$ & $0,55^{b}$ & $378,34^{b}$ \\
\hline 40 & 60 & $31,7^{c}$ & $10,71^{b}$ & $14,65^{a}$ & $44,93^{b}$ & $2,82^{b}$ & $1625^{b}$ & $899,67^{b}$ & $0,55^{b}$ & $354,41^{b}$ \\
\hline 30 & 70 & $32,07 c$ & $10,30^{a}$ & $14,63^{a}$ & $41,93^{b}$ & $2,95^{b}$ & $1620^{b}$ & $905,00^{b}$ & $0,55^{b}$ & $340,59^{b}$ \\
\hline 20 & 80 & $32,2^{c}$ & $9,74^{a}$ & $14,64^{a}$ & $38,90^{a}$ & $3,02^{b}$ & $1545^{a}$ & $920,5^{c}$ & $0,59 c$ & $326,32^{a}$ \\
\hline
\end{tabular}

Les valeurs indiquées représentent la moyenne de trois déterminations $(n=3)$. Dans chaque colonne, les valeurs affectées de lettres différentes sont significativement différentes à $P \leq 0,05$. Celles affectées de lettres identiques ne le sont pas. 
En fonction des différents types de gâteau, les informations nutritives pour $100 \mathrm{~g}$ sont données dans le tableau 3. Dans ce tableau, on observe que le gâteau de banane plantain contient moins d'amidon et de glucides totaux que les autres types de gâteaux, mais il a une humidité plus élevée. La valeur énergétique est peu élevée, comparativement au gâteau ordinaire, type cake, et au gâteau au chocolat.

Tableau 3: caractéristiques nutritives pour $100 \mathrm{~g}$ de gâteau de plantain 30/70 et de divers gâteaux.

\begin{tabular}{lcccc}
\hline $\begin{array}{l}\text { Composition } \\
\text { nutritive }\end{array}$ & $\begin{array}{c}\text { Gâteau de banane } \\
\text { plantain 30/70 }\end{array}$ & Gâteau de Savoie* & Gâteau type cake* & $\begin{array}{c}\text { Gâteau au } \\
\text { chocolat }^{*}\end{array}$ \\
\hline Protéines (g) & 10,3 & 10,3 & 6,1 & 4,2 \\
Glucides (g) & 41,93 & 50,7 & 49,9 & 55,1 \\
Sucres (g) & 30,2 & 30,1 & 26,4 & 35 \\
Amidon (g) & 11,73 & 20,6 & 17,4 & 16 \\
Lipides (g) & 6,33 & 6,5 & 20,4 & 22,5 \\
Eau (g) & 32,2 & 30 & 21,3 & 14,5 \\
Calorie (kcal) & 340,59 & 302 & 411 & 445 \\
\hline
\end{tabular}

* Source : table de composition nutritionnelle CIQUAL 2008

Evaluation organoleptique des gâteaux des différentes formulations : Le résultat de l'évaluation organoleptique des différents gâteaux est présenté dans le tableau 4. Ces résultats indiquent une préférence des dégustateurs pour les formules $50 / 50$, $40 / 60$ et $30 / 70$, par rapport à celle des gâteaux contenant $75 \%$ de farine de blé et $25 \%$ de banane plantain d'une part et $20 \%$ de farine de blé et $80 \%$ de banane plantain d'autre part. Des différences significatives, des points de vue de la couleur, de l'aspect, de l'arôme, du goût et de la texture ont été observées entre ces deux groupes de produits, selon le test de Newman-Keuls au seuil de $5 \%$. La moyenne des notes de l'appréciation du critère aspect est dans la catégorie "assez bon" et "bon". Les plus hautes moyennes ont été obtenues avec les formulations $50 / 50,40 / 60$ et $30 / 70$. La plus petite moyenne a été attribuée à la formulation 20/80. Les résultats de l'appréciation de l'arôme montrent que les moyennes augmentent quand le taux de substitution de la farine de blé avec la purée de banane plantain augmente. Des différences significatives, au seuil de $5 \%$, ont été observées, entre les formulations $100 \%$ farine de blé, $75 / 25$ et les formulations $60 / 40,50 / 50,40 / 60,30 / 70$ et $20 / 80$. La plus forte moyenne $(4,4)$ a été obtenue ave les formulations 40/60 et 30/70. L'évaluation du goût des gâteaux a montré que les moyennes des notes augmentent de la formulation $100 \%$ à $30 / 70$ et se situaient entre 3,5 et 4,3 avec les plus fortes moyennes $(4,25$ et 4,3$)$ pour $40 / 60$ et $30 / 70$

Tableau 4: Evaluation organoleptique des gâteaux en fonction des différentes formulations

\begin{tabular}{cccccccc}
\hline \multicolumn{2}{c}{ Mélange } & $\begin{array}{c}\text { Couleur de la } \\
\text { croute }\end{array}$ & $\begin{array}{c}\text { Couleur de la } \\
\text { mie }\end{array}$ & aspect & arôme & goût & texture \\
\cline { 1 - 4 } $\begin{array}{c}\text { Farine } \\
(\%)\end{array}$ & $\begin{array}{c}\text { Plantain } \\
(\%)\end{array}$ & & & & & & \\
\hline 100 & 0 & $2,5^{\mathrm{a}}$ & $2,6^{\mathrm{a}}$ & $3,1^{\mathrm{a}}$ & $2,8^{\mathrm{a}}$ & $3,5^{\mathrm{b}}$ & $3,9^{\mathrm{b}}$ \\
75 & 25 & $2,5^{\mathrm{a}}$ & $2,6^{\mathrm{a}}$ & $3,1^{\mathrm{a}}$ & $2,8^{\mathrm{a}}$ & $3,5^{\mathrm{b}}$ & $3,9^{\mathrm{b}}$ \\
60 & 40 & $2,8^{\mathrm{a}}$ & $3,8^{\mathrm{b}}$ & $3,5^{\mathrm{b}}$ & $3,1^{\mathrm{a}}$ & $3,8^{\mathrm{b}}$ & $4,1^{\mathrm{b}}$ \\
50 & 50 & $3,9^{\mathrm{b}}$ & $4,1^{\mathrm{b}}$ & $4,1^{\mathrm{b}}$ & $3,5^{\mathrm{b}}$ & $3,9^{\mathrm{c}}$ & $4,2^{\mathrm{b}}$ \\
40 & 60 & $4,1^{\mathrm{b}}$ & $4,3^{\mathrm{b}}$ & $4,5^{\mathrm{b}}$ & $4,4^{\mathrm{b}}$ & $4,3^{\mathrm{c}}$ & $4,5^{\mathrm{b}}$ \\
30 & 70 & $4,2^{\mathrm{n}}$ & $4,3^{\mathrm{b}}$ & $4,5^{\mathrm{b}}$ & $4,4^{\mathrm{b}}$ & $4,25^{\mathrm{c}}$ & $4,5^{\mathrm{b}}$ \\
20 & 80 & $2,6^{\mathrm{a}}$ & $3,1^{\mathrm{c}}$ & $2,3^{\mathrm{a}}$ & $3,5^{\mathrm{b}}$ & $2,8^{\mathrm{a}}$ & $2,9^{\mathrm{a}}$ \\
\hline
\end{tabular}

Les valeurs indiquées représentent la moyenne de trois déterminations $(n=3)$. Dans chaque colonne les valeurs affectées de lettres différentes sont significativement différentes à $P \leq 0,05$. Celles affectées de lettres identiques ne le sont pas.

\section{DISCUSSION}

La pulpe banane de plantain mûre a été utilisée pour fabriquer des gâteaux en y ajoutant de la farine et les autres ingrédients habituellement utilisés pour les produits de pâtisserie, dont les œufs, la matière 
grasse et la levure chimique. Pour déterminer les proportions de purée de pulpe de banane plantain et de farine à mettre en œuvre pour l'obtention d'un produit dont la qualité se rapproche de celle des gâteaux ordinaires, différents mélanges ont été testés. L'analyse de ces produits, issus des mélanges contenant 25 à $80 \%$ de plantain, a montré une augmentation des taux d'humidité et de sucres tandis que ceux des protéines, de l'amidon et des glucides totaux baissent. Les taux des matières grasses et de cendres sont restés constants. Cela s'expliquerait d'une part, par le fait que nous avons travaillé avec les mêmes quantités des ingrédients, autres que la purée de plantain et la farine pour tous les mélanges et, d'autre part, parce que la pulpe de banane plantain bien mûre, contient très peu d'amidon, comme l'ont montré Happi Emaga et al. (2007). Ainsi, quand la quantité de purée de banane plantain augmente, la teneur en amidon et en protéines diminue, alors que celle des sucres totaux augmente. Cette observation était prévisible, car la banane plantain est un produit énergétique, comme l'igname et le manioc, qui ont aussi une faible teneur en protéines, de l'ordre de 1 à $2 \%$ (Kahlon et Smith, 2007). Par ailleurs, la banane plantain mûre contient environ $8 \%$ d'amidon et $20 \%$ de sucres totaux (Von Loesecke, 1950; Ketiku, 1973). Nous avons également observé que l'augmentation du pourcentage de la purée conduit à un produit relativement moins volumineux, car la purée de banane plantain n'est pas panifiable. Elle ne contient pas de gluten (Sabanis et Tzia, 2009 ; Sciarini et al., 2010 ; Oduro-Yeboah et al., 2012 ; Melito et Farkas, $2013)$; il s'en suit une mauvaise rétention du dioxyde de carbone $\left(\mathrm{CO}_{2}\right)$ produit par la levure chimique, qui s'échappe et entraine un affaissement de la structure du gâteau avant la fin de la cuisson. Ce phénomène est plus prononcé avec la formulation $20 \%$ de farine et $80 \%$ de purée de pulpe de banane plantain, qui donne un gâteau compacte présentant de grandes alvéoles irrégulières, avec un volume dont la mesure est significativement différente des autres gâteaux. Au cours de la cuisson des gâteaux le gluten contenu dans la farine coagule sous l'effet de la chaleur, se solidifie et sert alors de structure rigide, qui maintient le volume développé en place. Ainsi, l'augmentation du taux de substitution de la farine entraine une diminution de celui du gluten, ce qui explique la différence de volume par rapport au gâteau fait avec $100 \%$ de farine, bien que cette diminution ne soit pas significative sauf pour la formulation 20/80. Ces observations sont en accord avec celles de Gomez et al. (2008), Turabi et al. (2008) et de Vitali et al. (2009) qui ont conduit des essais pour fabriquer des gâteaux à moindre coût, en substituant partiellement la farine de blé par de la farine de légumineuse tel que le haricot. Nous avons aussi observé une augmentation du poids et de la densité des gâteaux en fonction de la quantité de purée de pulpe de banane plantain dans le mélange. Cela serait certainement dû à la forte teneur en eau de la pulpe de banane plantain $(67 \%)$. II est probable que la capacité d'hydratation de la farine ait été accrue et partant, pour le même temps et la même température de cuisson, le gâteau conserve plus d'eau comme le confirme le taux d'humidité de $32 \%$ du gâteau fait avec le mélange purée de banane plantain et farine. Le taux d'humidité qui augmente en fonction du taux de substitution de la farine avec la purée de banane plantain a eu pour avantage de donner en fin de cuisson un produit plus mou avec une meilleure présentation et un caractère fondant à la dégustation comparativement aux gâteaux confectionnés avec 75 et $100 \%$ de farine de blé. L'augmentation du taux d'humidité peut être associée à celle du taux de fibre comme l'ont montré Elleuh et al. (2011) et Maneju et al. (2011). Toutefois, l'augmentation du taux d'humidité peut entrainer, une réduction du temps de conservation, à température ambiante. Dans ces conditions la conservation au réfrigérateur s'avère nécessaire. La qualité des produits de pâtisserie dépend de plusieurs facteurs tels que les ingrédients utilisés pour la préparation de la pâte, l'aération et les conditions d'élaboration. C'est donc de la qualité de la pâte que dépend la qualité du produit final caractérisée par le volume et l'uniformité de la structure de mie (Yang et Foegeding, 2010). L'évaluation sensorielle a été conduite en notant sur une échelle de 1 à 5 les caractères tels que la couleur de la croûte, la couleur de la mie, l'aspect général, l'arôme et la texture. Les résultats de cette évaluation montrent que la substitution avec 40,50 , 60 et $70 \%$ de purée de banane plantain donne une meilleure acceptabilité pour ces différents types de gâteaux. Ainsi, la préférence des consommateurs s'est portée sur ces produits, certainement, à cause des caractères particuliers conférés par la purée de pulpe de banane plantain bien mûr. La couleur de la croûte des gâteaux élaborés avec la purée de pulpe de banane plantain et la farine est plus foncée que celle des gâteaux faits avec 75 et $100 \%$ de farine, probablement à cause de la couleur jaune orangé et ne peut être attribuée seulement à la caramélisation des sucres. Quant à la couleur de la mie, qui présente une couleur jaune orangé, elle a fait la préférence des consommateurs, certainement à cause de l'attirance à cette couleur, contrairement au gâteau avec $100 \%$ de farine qui est de couleur jaune claire. Les caractéristiques nutritives du gâteau de banane plantain et de quelques gâteaux vendus sur 
le marché indiquent que le gâteau de banane plantain est moins énergétique et peut fournir 340,59 $\mathrm{kcal} / 100 \mathrm{~g}$ contre 411 et $445 \mathrm{kcal} / 100 \mathrm{~g}$ respectivement pour le gâteau type cake et le gâteau au chocolat. Cela pourrait s'expliquer par le fait que le gâteau de banane plantain a des taux de glucides

\section{CONCLUSION}

L'objectif de cette étude étant la réduction des pertes post récolte, la diversification de l'utilisation de la banane plantain peut aider à promouvoir une grande utilisation de ce produit. Ainsi, l'incorporation d'un maximum de pulpe de banane plantain mûre dans les produits de pâtisserie peut aussi aider à réduire les pertes après le murissement pendant la période de grandes productions. Pour ces raisons, la formule $30 / 70$, soit le mélange de $30 \%$ de farine et de $70 \%$ de purée de banane plantain, a été retenue comme formule type pour la préparation du gâteau de banane plantain. Les gâteaux préparés avec $70 \%$ de substitution de la farine de blé par la purée de banane plantain ont de bonnes caractéristiques sensorielles et peuvent être considérés comme produits compétitifs en comparaison avec les produits similaires vendus dans le commerce. La préparation de ces produits présente un avantage certain, car ils sont faits avec des ingrédients naturels sans sucre ajouté et ont une bonne qualité gustative. La pulpe de banane plantain bien mûr séchée et

\section{REFERENCES BIBLIOGRAPHIQUES}

Adopo NA, 1991. Réduction des pertes post récolte des bananes plantains. FAO, bourse de recherche André Mayer Rome (Italie) : Rapport final, $132 \mathrm{p}$.

Akesowan A, 2009. Quality of reduced-fat chiffon cakes prepared with erythritol-sucralose as replacement for sugar. Pakistan journal of nutrition 8 (9): 1383-1386.

Bonet A, Oliete B, Rosell CM, Pando V, Fernandez $E, 2008$. Study on cake quality made of wheat-chickpeas flour blends. Food Science and technology 41(9): 1701-1709.

Chaiya B, Pongsawatmanit R, 2011. Quality of batter and sponge cake prepared from wheat tapioca flour blends. Kasetsart Journal 4: 305-313.

Gomez MB, Oliete CM, Rosel CM, Pondo V, Fernandez E, 2008. Studies on cake quality made of wheat -chickpea flour blends. LWT. Food Science and Technology 41: 17011709.

Gupta M, Sing Bawa A, Semwal AD, 2011. Effect of barley flour blending on functional baking and organoleptic characteristics of high totaux et de lipides inférieurs à celui des autres types de gâteaux que sont le gâteau de Savoie, le gâteau type cake et le gâteau au chocolat, malgré la teneur en protéines de 10,3 \% qui indique que le gâteau de plantain est aussi nutritif que les gâteaux déjà commercialisés.

conditionnée peut être conservée sur plusieurs mois ce qui peut permettre de réduire les pertes post récolte de la banane plantain surtout après maturation complète. Le profil microbiologique de la pulpe de plantain mûr reste constant au cours de la conservation pendant 3,6 et 12 mois, à température ambiante; ce qui indique que le conditionnement dans le sachet de polyéthylène de 80 microns d'épaisseur et les conditions de stockage sont satisfaisants pour garantir une bonne qualité sanitaire; ainsi, l'utilisation de la pulpe séchée et conservée peut être considérée comme sans danger pour fabriquer des produits de pâtisserie. Les résultats de nos travaux permettent de recommander que la technologie utilisant la purée de pulpe de banane plantain bien mûr soit encouragée pour que les industries locales l'utilisent dans la fabrication des produits de pâtisserie de haute qualité. La production de la pulpe séchée peut se faire à moindre coût vue qu'elle se fait à l'aide du soleil.

husks. Journal of food processing and preservation 35: 46-63.

Hemeda HM, Mohamed EF, 2010. Functional attribute of chickpea and defatted soybean flour blends on quality characteristics of shortening cake. European journal of applied sciences 2 (2): 44-50.

Hussein EA, El-Beltagy AE, Gaafar AM, 2011. Production and quality evaluation of low calorie cake. American journal of food technology 6 (9): 827-834.

Ketiku AO, 1973. Chemical composition of unripe (green) and ripe plantain (Musa AAB). Journal of Science and Agriculture 24: 703707.

Mandala I, Daouaher M, 2005. The sensory attributes of cakes containing large numbers of low sugar raisins and a trained sensory panel. International Journal of food Science and Technology 40 (7): 759-769.

Melito H, Farkas BE, 2013. Physical properties of gluten-free donuts. Journal of Food Quality. 36: 32-40. 
Mepba HD, Eboh L, Nwojigwa SU, 2007. Chemical composition, functional and baking properties of wheat-plantain composite flours. African Journal of Food Agriculture Nutrition and Development 7 (1) 1-22.

Ndife J, Abdulraheem LO, Zakari UM, 2011. Evaluation of the nutritional and sensory quality of functional breads produced from whole wheat and soya bean flour blends. African Journal of Food Science 5 (8): 466472.

Oduro-yeboah C, Onwulata C, Tortoe C, ThomasGahring A, 2012. Functional properties of plantain, cowpea flours and oat fiber in extruded products. Journal of Food Processing and Preservation 1: 1- 9.

Sabanis D, Tzia C, 2009. Effect of rice, corn and soy flour addition on characteristics of bread produced from different wheat cultivars. Food bioprocess and Technology 2(1): 6879.

Scarini LS, Ribota PO, Leon AE, Perez GT, 2010. Influence of gluten-free their and their mixtures on batter properties and bread quality. Food bioprocess and Technology 3(4): 577-595.

Turabi EG, Sumnu G, Sahin S, 2008. Rheological properties and quality of rice cakes formulated with different gums and an emulsifier blend. Food Hydrocolloid 22(2): 305-312.

Von Loesecke 1950. Chemical change during ripening of bananas. Chemistry Physiology and Technology 4: 67-118.

Yang X, Foegeding EA, 2010. Effect of sucrose on egg white protein and whey protein Isolate foams: factors determining properties of wet and dry foams (cakes). Food Hydrocolloid 24: $227-238$. 